\title{
Empirische Grundlagen der Wirtschaftspolitik
}

\section{Christian Keuschnigg}

Sind inklusives Wachstum und wirtschaftliche Sicherheit möglich? Welche Rahmenbedingungen muss der Staat setzen und welche Reformen auf den Weg bringen, damit der Wohlstand nachhaltig zunimmt? Welche Anstrengungen und Investitionen müssen Familien und Unternehmen tätigen? Wie können die schwachen Gruppen der Gesellschaft angemessen teilhaben? Solche Fragen dominieren die Wirtschaftspolitik. Dabei gibt es weder in der Politik noch in der Wirtschaft einen endgültigen Erfolg. Globalisierung, Innovation und struktureller Wandel stellen das Erreichte immer wieder in Frage.

\section{Forschung für die wirtschaftspolitische Praxis}

Eine erfolgreiche Wirtschaftspolitik steht auf festen theoretischen und empirischen Fundamenten. Eine Wirtschaftspolitik ohne Theorie wäre Aktionismus ohne Ziel und Plan. Eine Politik ohne gesicherte empirische Grundlagen wäre Spekulation. Die Erreichung der Politikziele hängt davon ab, dass die getroffenen Massnahmen verlässlich vorhersehbare Wirkungen entfalten. Deshalb sind die Evaluationsstudien der empirischen volkswirtschaftlichen Forschung so ausserordentlich wichtig. Um Reformen auf den Weg zu bringen, investieren die Entscheidungsträger persönliche Reputation und politisches Kapital und müssen sich meist zu schwierigen Kompromissen durchringen. Wie könnte es sich für einen Wirtschaftspolitiker auszahlen, sich für Reformen zu engagieren, wenn die erwarteten positiven Wirkungen ungewiss erscheinen oder die geschätzten Wirkungen zwar statistisch hoch signifikant, aber quantitativ vernachlässigbar sind? Die in

C. Keuschnigg $(\bowtie)$

FGN, Universität St. Gallen, St. Gallen, Schweiz

E-Mail: christian.keuschnigg@unisg.ch

C. Keuschnigg (Hrsg.), Inklusives Wachstum und wirtschaftliche Sicherheit, https://doi.org/10.1007/978-3-658-21344-2_1 
der Wirtschaftspolitik postulierten Zusammenhänge sollen daher nicht nur statistisch signifikant, sondern auch quantitativ bedeutsam sein.

Wie kann die ökonomische Forschung für die Wirtschaftspolitik nutzbar werden? Dazu braucht es einen funktionierenden Wissenstranfer in die Praxis. Entscheidungsträger haben weder die Zeit noch die fachliche Kompetenz, die Entwicklungen in den spezialisierten Fachzeitschriften zu verfolgen. Sie verfügen über einen erfahrenen und fachlich kompetenten Mitarbeiterstab, der die Forschungsergebnisse aufbereitet, damit sie in den Entscheidungsprozess einfliessen können. Sie sollten aber idealerweise ihre eigene Richtlinienkompetenz schärfen, indem sie sich die wichtigsten Einsichten der neueren Forschung aneignen und sich ein eigenständiges und unabhängiges Urteil bilden. Ebenso muss sich in der Demokratie die Öffentlichkeit ein informiertes Bild von grundlegenden wirtschaftlichen Zusammenhängen machen, um die Politik kontrollieren und informierte Wahlentscheidungen treffen zu können. Mit der populären und allgemein zugänglichen Aufbereitung empirischer Forschungsbeiträge kann die Volkswirtschaftslehre den Wissenstransfer in die Praxis befördern.

Eine Schwierigkeit in der Nutzung der empirischen Forschung besteht darin, dass einzelne Arbeiten oft nur ein punktuelles Ergebnis bringen und häufig kein endgültiges Urteil erlauben. Je nach untersuchter Zeitperiode und je nach spezifischem institutionellem Hintergrund liefert die empirische Forschung eine grosse Bandbreite von Ergebnissen. Einzelne Forschungsbeiträge unterscheiden sich zudem im methodischen Anspruch, in der Qualität der quantitativen Analyse und daher in der Glaubwürdigkeit der Ergebnisse. In vielen Fällen lässt sich anhand einer kritischen Literaturübersicht oder mittels Meta-Studien ein breit abgestützter Konsens ableiten. Dieser Sammelband beschreitet jedoch einen anderen Weg, der an die universitäre Lehre anknüpft. Auch die Volkswirtschaftslehre an der Universität steht vor der Herausforderung, den Studierenden, den Alumnis, den Entscheidungsträgern der Wirtschaftspolitik und der interessierten Öffentlichkeit einen gesicherten Bestand des empirischen Wissens zu vermitteln.

Um zu verlässlichen Ergebnissen der volkswirtschaftlichen Spitzenforschung zu gelangen, erfolgt die Auswahl der Forschungsbeiträge in diesem Band nach drei Kriterien. Erstens stammen die Arbeiten aus den führenden Fachzeitschriften. Sie unterliegen damit einem harten Auswahlprozess mit einer überaus strengen Qualitätskontrolle. Die besten Fachjournale akzeptieren nur fünf bis zehn Prozent der eingereichten Beiträge, die zudem in einem aufwendigen Begutachtungs- und Überarbeitungsprozess noch weiter verbessert werden. Der Wettbewerb ist ähnlich intensiv wie in Sport und Kultur, wo der Wettbewerb zu Spitzenleistungen anspornt, oder in der Privatwirtschaft, wo nur die innovativen Unternehmen rasch wachsen können und mangelnde Leistung den Untergang 
bedeutet. Zweitens stellt die Auswahl auf das Ansehen der Wissenschaftler in der Fachwelt ab, die ihre Reputation mit einer Vielzahl von Forschungserfolgen geschaffen haben und daher in ihrem Fachgebiet tonangebend sind. In den Spitzenjournalen vergehen oft mehrere Jahre bis zur tatsächlichen Publikation der Beiträge. Daher berücksichtigt der Sammelband auch ganz neue Forschungsarbeiten von führenden Ökonomen, die bereits als Diskussionspapiere renommierter Universitäten und Forschungsnetzwerke zugänglich, aber noch nicht publiziert sind. Das dritte Auswahlkriterium ist die wirtschaftspolitische Relevanz und der Anwendungsbezug.

Die Ergebnisse der empirischen Forschung erfahren die Studierenden in spezialisierten Lehrveranstaltungen. Dabei kommt die themenübergreifende Darstellung, welche zentrale Zusammenhänge und Querbezüge aufzeigt, oft zu kurz. Solche Querbezüge sind gerade auch für die wirtschaftspolitische Praxis wichtig. Wie sonst könnte es möglich sein, die Komplementarität oder Rivalität wirtschaftspolitischer Strategien zu erkennen? Die Wirtschaftspolitik handelt oft punktuell und isoliert. Damit droht der Gesamtzusammenhang aus dem Blick zu geraten. Ein Beispiel dafür sind die regulatorischen Initiativen für strengere Eigenkapital- und Liquiditätsstandards für Banken, um die Stabilität des Finanzsystems zu stärken. Dabei wird übersehen, dass das Steuersystem einen starken Anreiz zur Überschuldung setzt und der Staat selbst zur Instabilität beiträgt. Es ist ein Widerspruch, dass der Staat mit der Abzugsfähigkeit von Zinsen auf Fremdkapital und Immobilienkrediten die Überschuldung von Haushalten, Unternehmen und der Banken fördert, und dann mit Finanzmarktregulierung wieder das Gegenteil anstrebt, nämlich weniger Verschuldung und mehr Eigenkapital. Solche Widersprüche entstehen, wenn wichtige Querbezüge vernachlässigt und daher die Komplementarität oder Rivalität unterschiedlicher wirtschaftspolitischer Handlungsfelder nicht erkannt werden.

Der vorliegende Sammelband stellt empirische Forschungsarbeiten auf unterschiedlichen Gebieten thematisch zusammen und will in diesem Überblickskapitel einen wirtschaftspolitischen Gesamtzusammenhang herstellen. Angesichts der Breite des Fachs bleibt die Auswahl der nachfolgenden Beiträge thematisch selektiv und lückenhaft. Dennoch beleuchten sie zentrale Handlungsfelder der Wirtschaftspolitik und reichen von der Rolle des Staates bis zur Stabilität des Finanzsystems.

\section{Die Rolle des Staates}

Der Staat setzt die wirtschaftlichen und gesetzlichen Rahmenbedingungen, organisiert öffentliche Leistungen, welche die Lücken im privatwirtschaftlichen Angebot schliessen, sorgt für soziale Sicherheit und eine ausgewogene Verteilung, und 
finanziert sich mit Steuern und Gebühren. Er handelt im Auftrag der Bürgerinnen und Bürger und soll die Interessen der grossen Mehrheit bedienen. Dabei gibt es höchst unterschiedliche Vorstellungen über die Staatsaufgaben. Am Ende gelten die Entscheidungen jedoch für alle gleich. Wie können die Wähler sicher sein, dass die Ergebnisse der Politik tatsächlich im Sinne der grossen Mehrheit ausfallen? Drohen das Streben nach politischer Macht und Prestige und die Bedienung mächtiger Interessensgruppen den Wählerauftrag zu verfälschen? Der erste Beitrag zeigt anhand empirischer Evidenz aus der Schweiz, wie direkte Demokratie und fiskalischer Wettbewerb zwischen Gemeinden und Kantonen die Politik disziplinieren können. Die beiden Mechanismen ergänzen sich. In jenen Regionen, wo der fiskalische Wettbewerb nur schwach ausgeprägt ist, ist die direkt demokratische Kontrolle besonders wichtig. Wenn die Wähler in einer Gemeinde selbst über die Höhe der Einkommensteuer abstimmen können, zahlen sie um $3 \%$ tiefere Steuern als in Gemeinden mit nicht direkt demokratischer Verfassung. Fehlt es an direkt demokratischen Einflussmöglichkeiten, dann wirkt der Steuerwettbewerb disziplinierend.

Der Staat finanziert sich mit Steuern und Gebühren. Gebühren sind der Preis für eine individuell zurechenbare Leistung. Steuern sind notwendig, wenn öffentliche Leistungen wie Rechtssicherheit, Verteidigung und innere Sicherheit, Grundlagenforschung, oder Umweltqualität allen gleich zur Verfügung stehen, und wenn Umverteilung zu finanzieren ist. Ein einzelner Bürger alleine kann aber weder Umfang und Verwendung öffentlicher Ausgaben noch die Steuerfinanzierung bestimmen. In Zeiten knapper öffentlicher Budgets ist meist für gemeinnützige Zwecke, die vielen Bürgern wichtig sind, nicht genug Geld da. Erfolgreiche und vermögende Personen sind oft motiviert, der Gesellschaft etwas zurückzugeben und fördern mit grossen Spenden ihnen besonders wichtig erscheinende gemeinnützige Zwecke. Auch viele Bürger mit wenig Einkommen spenden kleine Beträge. Wie können Staat und gemeinnützige Organisationen freiwillige Beiträge für gemeinnützige Zwecke mobilisieren? Steueranreize oder staatliche Zuschüsse zum privaten Spendenaufkommen regen die Spendentätigkeit an. Sinken Dank steuerlicher Abzugsfähigkeit die privaten Kosten der Spendenfinanzierung um $1 \%$, dann steigt das Spendenaufkommen um mehr als $1 \%$. Die Bürger spenden am ehesten dort, wo die staatliche Finanzierung zu kurz greift, und enthalten sich umso mehr, wenn der Staat selbst die Finanzierung ausweitet. Ein Euro mehr an öffentlichen Ausgaben für einen gemeinnützigen Zweck reduziert das Spendenaufkommen um 50 Cents.

Steuern finanzieren den Löwenanteil der Staatsausgaben. Das Steuersystem soll einfach, fair und leistungsfreundlich sein. Da die Staatsausgaben von allen gemeinsam finanziert werden, steht der individuellen Steuerzahlung keine persönlich 
zurechenbare Gegenleistung gegenüber. Steuern mindern daher die Leistungsbereitschaft und bremsen das Wachstum. Die Steuerzahler weichen der Besteuerung aus und versuchen, die Last auf andere zu überwälzen. Das führt zu schwierig abzuschätzenden Verteilungseffekten und erschwert die Verteilungspolitik. Der dritte Beitrag fasst neue Forschungsergebnisse aus den U.S.A. zusammen, wonach z. B. eine Senkung der lokalen Gewinnsteuerbelastung um $1 \%$ die Zahl der lokalen Unternehmen während 10 Jahren um rund 3 bis $4 \%$ erhöht. Das Umgekehrte gilt bei einer Steuererhöhung. Je mehr die Unternehmen ihre Investitionen zurücknehmen oder abwandern, desto ungünstiger entwickeln sich Löhne und Immobilienpreise in der Region. Die Last der Unternehmenssteuer wird demnach zu $40 \%$ von den Eigentümern, zu 30-35 \% von den Immobilienbesitzern und zu 25-30 \% von den Arbeitnehmern getragen. Es ergeben sich zwei Schlussfolgerungen. Die Besteuerung hat im wachsenden Steuerwiderstand ihre Grenzen, und die Verteilungspolitik muss das Endergebnis nach allen Anpassungsvorgängen im Blick haben.

Ein besonders schwer $\mathrm{zu}$ kontrollierende Form der Steuerausweichung ist die internationale Gewinnverschiebung multinationaler Unternehmen, z. B. mittels Verlagerung von Patenten, steuersparender Festsetzung von konzerninternen Lizenzgebühren und Verrechnungpreisen oder interner Kreditvergabe. Um Steuern zu sparen, können Multis z. B. den F\&E-Aufwand in Ländern mit hoher Steuerbelastung tätigen, aber die Patente auf Standorte in Niedrigsteuerländer übertragen, um dort die Patenterträge besonders niedrig zu versteuern. Das bringt die Hochsteuerländer unter Druck. Der vierte Beitrag berichtet von Forschungsergebnissen, wonach eine Erhöhung des Steuersatzes auf Patenteinnahmen um zehn Prozentpunkte die Anzahl von Patentanmeldungen in diesem Land um $35 \%$ verringert. Dabei kommt es nicht nur auf die Steuerbelastung im Inland an. Ein Land verliert auch dann Patente, wenn das Ausland die effektive Steuerbelastung auf Patenterträge senkt.

Nicht jede Steuerausweichung ist unerwünscht. Eine Staatsaufgabe ist die Beseitigung von Marktstörungen. Wenn die privaten Akteure gesellschaftlich relevante Erträge und Kosten teilweise nicht berücksichtigen, dann führt das private Streben nach Einkommen und Gewinn zu ,Fehlentscheidungen ' aus gesellschaftlicher Sicht, die es zu korrigieren gilt. Ein Beispiel ist die Neigung zur Überschuldung, welche die Finanzstabilität gefährdet und allen Marktteilnehmern schadet. Eine der Ursachen für die Überschuldung ist die steuerliche Bevorzugung des Fremdkapitals. Die steuerliche Gleichstellung von Fremd- und Eigenkapital sollte eine positive Verhaltensreaktion auslösen und dazu beitragen, die Überschuldung zu verringern und mit mehr Eigenkapitalbildung die Finanzstabilität zu stärken. Mit dieser Absicht hat Belgien die steuerliche Abzugsfähigkeit einer Eigenkapitalverzinsung beschlossen. 
Der fünfte Beitrag berichtet von Forschungsergebnissen, wonach die Eigenkapitalquote der belgischen Banken nach der Reform um über $13 \%$ zugenommen hat. Vor allem die schwach kapitalisierten Banken, bei denen eine Stärkung des Eigenkapitals besonders dringlich war, haben überdurchschnittlich stark reagiert. Während die Eigenmittel stark gestiegen sind, hat das Volumen der Aktiva und Kredite sich kaum verändert. Die Reform unterstützt damit die Wirksamkeit der regulatorischen Mindeststandards und sorgt für mehr Krisenrobustheit im Bankensektor.

\section{Bildung und soziale Inklusion}

Reichtum und Armut entstehen durch Anstrengung und Fleiss ebenso wie durch Glück und Pech. Armut kann die Folge mangelnder Anstrengung und Vorsorge sein. Es kann sich aber niemand aussuchen, ob sie oder er in eine reiche Familie hineingeboren wird, reichlich mit Talent ausgestattet ist oder nicht, spektakulären Erfolg in einem unternehmerischen Beruf erzielt oder wegen Invalidität, unverschuldeter Insolvenz oder anderen Schicksalsschlägen in die Armut schlittert. Das Bedürfnis nach wirtschaftlicher Sicherheit begründet den Sozialstaat. Er soll unterschiedliche Chancen zu Beginn und während des ganzen Lebens ausgleichen (Umverteilung), einen angemessenen Wohlstand in allen Lebenslagen erhalten (Versicherung) und auch eine angemessene soziale Teilhabe ermöglichen. Der Sozialstaat besteht aus den Institutionen der Sozialversicherung, die mit Versicherungsbeiträgen persönliche Leistungsansprüche begründen, und aus dem Steuer- und Transfermechanismus, der Einkommen oben mit progressiven Steuern abschöpft und unten mit Ersatzleistungen aufstockt.

Eine nachhaltig hohe soziale Inklusion braucht vorbeugende und korrigierende Massnahmen. Eine vorbeugende Politik soll Ungleichheit und soziale Risiken erst gar nicht entstehen lassen, und setzt daher auf Bildung, fairen Wettbewerb und freien Zugang zu Unternehmertum und lukrativen Berufen. Ein leistungsfähiges Bildungswesen und weitgehende Vollbeschäftigung sind die wichtigsten vorbeugenden Investitionen, die den Sozialstaat vor Überbeanspruchung schützen. Dagegen korrigieren die Sozialversicherung und der Steuer-Transfer-Mechanismus eine entstandene Ungleichheit im Nachhinein. Es entstehen Anreizprobleme, welche den Umfang des Sozialstaats ausweiten und zunehmende Kosten verursachen. Eine grosszügige Absicherung mindert die Leistungsbereitschaft und das Vorsorgeverhalten, so dass mehr Menschen Leistungen in Anspruch nehmen und weniger zur Finanzierung beitragen. Diese Themen beleuchtet der Abschnitt über Bildung und soziale Inklusion aus verschiedenen Blickwinkeln.

Ungleichheit ist jedoch keine statische Angelegenheit und Armut kein Schicksal. Sozialer Aufstieg und Abstieg verändern die Position in der Gesellschaft, im Laufe des Lebens und in der Abfolge der Generationen. Wer heute arm ist und 
seine Chancen nutzt, bringt es morgen zu Wohlstand. So lautete der amerikanische Traum des Aufstiegs vom Tellerwäscher zum Millionär. Die Forschung zeigt jedoch, dass die U.S.A. heute nicht mehr das Land mit der höchsten sozialen Mobilität sind, wie der erste Beitrag des Abschnitts zusammenfasst. Die Wahrscheinlichkeit, dass Kinder aus einer Familie der $20 \%$ tiefsten Einkommen im Laufe des Lebens in die Top $20 \%$ aufsteigen, beträgt in den U.S.A. etwa 7,5\%. Dagegen liegen diese Aufstiegschancen in Kanada bei 13,4 und in Dänemark bei 11,4\%. Ghettobildung, Ungleichheit, Schulqualität, Sozialkapital und Familienstruktur beeinflussen die soziale Mobilität und können $76 \%$ der Unterschiede in den sozialen Aufstiegschancen zwischen den Regionen der U.S.A. erklären.

Das Bildungswesen, das speziell auch die Kinder aus bildungsfernen Elternhäuser erreicht, ist für Chancengleichheit und sozialen Aufstieg entscheidend. Leistungsfähige Schulen brauchen gutes Management, um die Produktivität zu steigern und mehr aus dem Steuergeld herauszuholen. Es gilt, das richtige Personal auszuwählen, klare Ziele vorzugeben, Leistungsanreize zu setzen, die Zielerfüllung zu kontrollieren und die Abläufe zu korrigieren, wenn die Leistung nicht stimmt. Der zweite Beitrag berichtet von einer Untersuchung von 1800 zufällig ausgewählten Schulen in acht Ländern, wonach moderne Managementmethoden wenig verbreitet sind und grosse Unterschiede zwischen den Schulen bestehen. Gutes Schulmanagement könnte die Leistungen der Schülerinnen und Schüler um 23 bis $42 \%$ einer Standardabweichung (als Mass für die Unterschiede zwischen den Schulen) verbessern. Es zeigt sich offensichtlich, dass staatliche Schulen über die beste Managementqualität verfügen, wenn sie autonom über Personal, Zulassung und Curriculum entscheiden können.

Die Sozialversicherung soll vor Einkommensverlusten schützen und wirtschaftliche Sicherheit vermitteln, kann aber unerwünschte und bisweilen überraschende Nebenwirkungen haben. Eine umfangreiche Versicherung mindert die Anreize zu vorbeugendem Verhalten, steigert die Häufigkeit der Schadensfälle und verteuert damit die Versicherung. Das zeigt sich auch im dritten Beitrag zur Arbeitslosenversicherung in Österreich. Von 1988 bis 1993 wurde zur Abfederung eines regional konzentrierten Strukturwandels in 28 von ca. 100 Bezirken die maximale Bezugsdauer des Arbeitslosengeldes für ältere Arbeitnehmer mit langer Beschäftigungsdauer um drei Jahre angehoben. Mit der Einführung des Programms stieg bei den geförderten Arbeitnehmern die Arbeitslosendauer um 43 Wochen an. Die längere Abwesenheit vom Arbeitsmarkt verringerte die Stellenkonkurrenz und erleichterte die Jobsuche der übrigen Arbeitslosen, die in der Folge ihre Arbeitslosigkeit um 4 Wochen verkürzen konnten.

Ein Kernthema der sozialen Inklusion ist die Vereinbarkeit von Beruf und Familie und die Gleichberechtigung der Frauen auf dem Arbeitsmarkt. Wie 
reagieren Haushalte und Arbeitgeber auf familienpolitische Massnahmen? Der vierte Beitrag fasst die empirische Evidenz zur Familienpolitik in den hoch entwickelten Ländern zusammen. Demnach kann z. B. eine bezahlte Elternzeit von drei Monaten die Beschäftigungsquote von Frauen um 3 bis $4 \%$ erhöhen, ohne ihre Löhne zu beeinträchtigen. Ein längerer Elternurlaub trägt jedoch nichts mehr zur Beschäftigungsquote der Frauen bei, senkt aber ihre Löhne um ca. $3 \%$. Am ehesten profitieren die gering qualifizierten Frauen, während ein längerer Berufsunterbruch die Arbeitsmarktchancen hoch qualifizierter Frauen beeinträchtigt. Eine wirksame familienpolitische Massnahme ist die frühkindliche Betreuung. Eine Erhöhung der Ausgaben für Kinderbetreuung um $1 \%$ des BIPs führt zu einem Anstieg der Frauenerwerbsquote von 3,6 Prozentpunkten.

Investitionen in die Kinderbetreuung bauen Ungleichheiten zwischen Männern und Frauen auf dem Arbeitsmarkt ab und regen zudem die Geburtenraten an. Die Fertilität der Frauen und die Chancen der Kinder auf eine entwicklungsfördernde familiäre Betreuung hängen jedoch von vielen Faktoren ab. Auch langanhaltende demographische Verschiebungen als Folge eines Männermangels dürften die Chancen der Frauen und Kinder nach dem zweiten Weltkrieg beeinträchtigt haben. Der fünfte Beitrag zeigt, dass von 1939 bis 1946 der Anteil unehelicher Geburten in den bayerischen Landkreisen von 9,2 auf 20,8\% angestiegen ist. Die Forscher schätzten unter Berücksichtigung vieler anderer Einflussgrössen, dass die Reduktion des Geschlechterverhältnisses um $1 \%$ zu einem Anstieg des Anteils ausserehelicher Geburten von 1,2\% in 1946 führte.

Frauen sind in leitenden Positionen stark untervertreten. Der Frauenanteil in den Führungspositionen grosser börsenkotierter Unternehmen lag 2013 in den U.S.A. bei tiefen $14 \%$, wie der sechste Beitrag berichtet. Dabei kann sich eine höhere Diversität in den Aufsichts- bzw. Verwaltungsräten durchaus positiv auf die Unternehmenswerte auswirken, indem Frauen wichtige fachliche Kompetenzen einbringen, die in reinen Männergremien fehlen. Dadurch kann sich die Qualität der Unternehmenskontrolle und strategischen Beratung durch den Verwaltungsrat verbessern. Die Forschungsarbeit zeigt tatsächlich, dass neu in den Verwaltungrat aufgenommene Frauen im Schnitt 0,53 zuvor nicht abgedeckte Kompetenzen einbringen, im Unterschied von nur 0,32 neuen Kompetenzen bei Männern.

Gesundheit fördert Lebensqualität und wirtschaftlichen Erfolg. Ein wesentlicher Aspekt sozialer Inklusion ist daher der Zugang zum Gesundheitswesen insbesondere für einkommensschwache Gruppen und eine rasche Behandlung hoher Qualität im Notfall. Andererseits ist gerade das Gesundheitswesen und dabei vor allem die stationäre Behandlung in Spitälern eine wichtige Ursache für einen starken Kostenanstieg. Es stellt sich die schwierige Frage, ob die intensivere und 
teurere Behandlung in den Spitälern mit einer besseren Versorgung von Patienten gerechtfertigt werden kann. Der siebte Beitrag zeigt, dass eine gute Erstbehandlung in Spitälern hohe Folgekosten vermeiden und sich durchaus auszahlen kann. Das Ergebnis einer ökonometrischen Untersuchung für die U.S.A. ist, dass eine Erhöhung der Spitalsausgaben pro Patient um US\$ 1800 die Sterberate der Patienten, welche per Ambulanz eingeliefert werden, um $10 \%$ von 37 auf 33,3\% verringert. Eine intensive Erstbehandlung ist zwar zunächst kostspielig, kann aber Einsparungen bei teuren Nachbehandlungen ermöglichen. Die Forscher finden daher, dass eine Zunahme der Kosten für die Erstbehandlung um $10 \%$ die medizinischen Gesamtkosten innerhalb des ersten Jahres nur um $6 \%$ steigert.

\section{Forschung und Innovation}

Die Wertschöpfungskette der Innovation beginnt mit der Grundlagenforschung und endet mit der kommerziellen Verwertung. In der Marktwirtschaft spielen Staat und private Unternehmen klar definierte Rollen. Die Grundlagenforschung ist eine klassische Staatsaufgabe, da das neue Wissen wie ein öffentliches Gut ohne besondere Einschränkungen allen zur Verfügung steht. Das neu geschaffene Wissen erleichtert wie andere produktive Vorleistungen die privaten F\&E-Investitionen. Die Erkenntnisse der Grundlagenforschung fliessen jedoch nicht automatisch in die Privatwirtschaft. Um sie für die kommerzielle Anwendung zu erschliessen, muss ein Wissenstransfer stattfinden, z. B. durch hoch qualifiziertes Personal, universitäre Patente und Neugründungen von jungen Technologieunternehmen. Dafür braucht es separate Investitionen und geeignete Anreize für Universitäten und Unternehmen.

Mit den privaten F\&E-Investitionen legen die Unternehmen den Grundstein für ihr Wachstum. Aber auch die private F\&E entfaltet unentgeltliche Vorteile für die gesamte Wirtschaft, weil die neuen Lösungen rasch bekannt werden oder durch Personalwechsel in andere Unternehmen getragen werden. Damit fällt die gesellschaftliche Ertragsrate deutlich höher aus als die rein private F\&E-Rendite. Das rechtfertigt eine staatliche Innovationsförderung, um die private Innovation auf das richtige Niveau zu heben. Dabei rechnet sich der Einsatz von Steuergeldern nur, wenn die staatliche Förderung sich nicht in Mitnahmeeffekten erschöpft, sondern die privaten F\&E-Investitionen ausreichend stark hebeln kann.

Um den Wissenstransfer aus den Universitäten voranzutreiben, müssen die Forscher Zeit und grossen Aufwand tätigen, um Patente zu entwickeln und spezielle Anwendungen für die Privatwirtschaft $\mathrm{zu}$ erschliessen. Diese Investitionen erfolgen nicht von alleine, weil sie zu Lasten der Kernaufgaben in Grundlagenforschung und universitärer Lehre gehen. Finanzielle Anreize können helfen, angemessene Investitionen für einen Wissenstransfer anzustossen und zur 
kommerziellen Nutzung der universitären Forschung beizutragen. Das zeigt sich im ersten Beitrag dieses Abschnitts zu den Auswirkungen einer Erfolgsbeteiligung von Professoren. Norwegen hat 2003 die vorher mögliche Beteiligung der Wissenschaftler an den finanziellen Erträgen ihrer Forschung abgeschafft und die Einnahmen in die Universitätshaushalte übertragen. In der Folge sank die Zahl universitärer Neugründungen von $24,7 \%$ vor der Reform auf $10,8 \%$ danach. Das ist ein Rückgang von $56 \%$. Die Reform senkte die Wahrscheinlichkeit, dass ein universitärer Forscher ein Unternehmen gründet, um ca. $63 \%$. Die gesamten Patentanmeldungen einer Universität gingen um $20 \%$ und jene eines universitären Forschers um $48 \%$ zurück. Diese Ergebnisse stützen die Erkenntnis, dass Eigentumsrechte - wie überall sonst - auch für die Ausrichtung der universitären Forschung wichtig sind.

Die private F\&E der Unternehmen schafft Wissen und Know-How, das nicht nur den eigenen Erfolg stärkt, sondern auch anderen Firmen nützt. Die zusätzlichen Erträge für andere rechtfertigen eine Innovationsförderung mit Steuererleichterungen, aber nur, wenn sie tatsächlich mehr Forschung anstossen. Den Auswirkungen steuerlicher F\&E-Förderung geht der zweite Beitrag nach. Grossbritannien hat 2008 die steuerliche Abzugsfähigkeit von F\&E-Ausgaben erhöht und den Kreis der anspruchsberechtigten KMU erheblich ausgedehnt. Heute können KMU $175 \%$ und Grossunternehmen $130 \%$ der F\&E-Ausgaben von der Steuer absetzen. Nach der Steuerreform hat sich die F\&E in den betroffenen Unternehmen ungefähr verdoppelt. Dabei ist der Effekt bei jenen Unternehmen stärker, die vorher schon F\&E betrieben haben. Die Steueranreize haben bei gleichbleibender Qualität der Innovationen die Zahl der Patente um $58 \%$ gesteigert. Der Anstoss ist, dass Steueranreize die privaten F\&E-Kosten absenken. Die Wissenschaftler schätzten, dass eine Absenkung dieser Kosten um $1 \%$ die F\&E-Investitionen um 2,6\% steigern können. Steueranreize können also sehr wirksam sein, private Innovation zu stärken.

Auch der dritte Beitrag geht der Wirksamkeit staatlicher Innovationsförderung nach. Die Forscherin nutzte Daten von 1995 bis 2013 des Small Business Innovation Research (SBIR) Programms des Energieministeriums. Das SBIR ist das grösste Förderprogramm der U.S.A., welches Subventionen an High-Tech Unternehmen vergibt. Die Förderung erfolgt in zwei Stufen. Zunächst bewerben sich Start-ups um eine wettbewerblich vergebene Subvention von US\$150.000. Wenn sie damit erfolgreich sind, können sie sich zwei bis drei Jahre später, wenn sie schon etwas älter sind, um eine Anschlussförderung von bis zu US\$ 1 Mio. bewerben. Ein Ziel des Programms ist es, privates Wagniskapital für junge Unternehmen zu mobilisieren. Die Wissenschaftlerin schätzt, dass nach einer Förderung die Wahrscheinlichkeit von Start-ups, Wagniskapital zu erhalten, sich sprunghaft 
von 10 auf $20 \%$ verdoppelt. Die Start-ups in der ersten Stufe des Programms halten im Durchschnitt 21 Patente. Eine Förderung erhöht die Zahl der Patentanmeldungen um etwa den Faktor 2,5. Die Anschlussförderung an die bereits etwas älteren Unternehmen hat dagegen keinen signifikanten Einfluss mehr auf die Finanzierung mit Wagniskapital und auf die Überlebensrate. Sie steigert jedoch die Zahl der Patente um das Doppelte. Das ist beträchtlich, aber deutlich weniger als bei der Frühförderung. Die Ergebnisse legen nahe, die Innovationsförderung vor allem auf junge innovative Start-ups zu konzentrieren.

\section{Unternehmen, Management und industrieller Wandel}

Innovation, gutes Management und hoch motiviertes Personal bringen die Unternehmen voran. Mit Innovation erwerben sie jenen Qualitäts- und Kostenvorsprung, mit dem sie im globalen Wettbewerb erfolgreich sind und ihre Stellung auf den Absatzmärkten ausbauen können. Daher investieren F\&E-intensive Unternehmen mehr, wachsen schneller und schaffen mehr Beschäftigung. Gut positionierte Unternehmen mit klaren Wettbewerbsvorteilen können ihren Arbeitnehmern mehr Jobsicherheit und Aufstiegschancen bieten. Bei mangelnder Innovation drohen Schrumpfung oder gar Marktaustritt, wenn die Nachfrager sich den Konkurrenten mit attraktiverem Angebot zuwenden. Innovationsgetriebenes Wachstum durch ,kreative Zerstörung“ verursacht daher einen starken Strukturwandel mit Gewinnern und Verlierern unter den Unternehmen und ihren Eigentümern wie auch bei den Arbeitnehmern.

Innovationsgetriebenes Wachstum ist bildungsintensiv und spreizt die Lohnschere, insbesonders wenn Qualifikation und Ausbildung der Arbeitenden nicht rasch genug nachziehen. Gering qualifizierte Arbeitnehmer geraten unter Druck, die gut ausgebildeten und hoch qualifizierten gewinnen. Innovation stärkt die Bildungsprämie und ist eine der Ursachen für grössere Ungleichheit. Spitzenmanager, Risikokapitalgeber und innovative Unternehmerpersönlichkeiten in den profitabelsten Unternehmen erwirtschaften stark steigende, aber auch sehr riskante Einkommen und tragen damit zu einem steigenden Einkommensanteil der Top-1-Prozent der Einkommen bei. Was sagt die empirische Forschung zum industriellen Wandel, den Folgen für die Einkommensverteilung und den Perspektiven der Arbeitnehmer?

Was tun Manager, um ein Unternehmen zum Erfolg zu führen? Sie entwickeln die Strategie, positionieren das Unternehmen gegenüber der Konkurrenz, sorgen für effiziente Abläufe in Produktion und Vertrieb, und setzen im Innovationsprozess auf profitable, zukünftige Geschäftsfelder. Der erste Beitrag zeigt, wie die Forschung anhand von Daten des ,World Management Surveys“ den Einfluss der Managementqualität auf den Unternehmenserfolg gemessen an Umsatz, Arbeitsproduktivität und 
Kapitalrendite ermittelt. Demnach kann ein Unternehmen seine Produktivität um 4,3\% steigern, wenn es seine Managementqualität um eine Standardabweichung (das entspricht einer Stufe auf der Skala von 1 bis 5) verbessert. Etwa die Hälfte des Effektes von besseren Managementmethoden auf die Umsätze wird durch die gezielte Beschäftigung von besser qualifizierten Mitarbeitern und Managern erzielt. Gut geführte Unternehmen neigen dazu, schlechte und wenig produktive Mitarbeiter öfter zu entlassen und vermehrt gut qualifizierte Mitarbeiter einzustellen. Eine hohe Managementqualität hat also einen ganz erheblichen Einfluss auf den Erfolg der Unternehmen und ihrer Belegschaft.

Die Unternehmen sind einem harten Wettbewerb mit hohem Erlösrisiko ausgesetzt, vor allem, wenn sie in innovativen, sich rasch wandelnden Branchen und auf weltweiten Absatzmärkten tätig sind. Während die Gewinne stark schwanken, bleiben Löhne und Beschäftigung vergleichsweise stabil. Die Unternehmen ,versichern' die Arbeitnehmer, wie der zweite Beitrag zeigt. Dabei bieten Familienunternehmen mehr Beschäftigungssicherheit und können ihre Angestellten in einer Krise eher überzeugen, mit vorübergehenden Lohnzugeständnissen die Beschäftigung zu erhalten. Wenn der Branchenumsatz um $10 \%$ einbricht, sinkt im Durchschnitt der Unternehmen der Reallohn um 0,5 bis $0,6 \%$, jedoch um 0,7 bis $0,9 \%$ in den Familienunternehmen. Dagegen sinkt die Beschäftigung in Nicht-Familienunternehmen mit 1,2 bis 1,9\% deutlich stärker als in Familienunternehmen, wo sie im Wesentlichen konstant bleibt. Die Erlöseinbrüche schlagen also nur zu einem geringen Teil auf Reallöhne und Beschäftigung durch.

Bringen Automatisierung und Digitalisierung den Mittelstand unter Druck? Der dritte Beitrag zeigt auf, dass die Automatisierung dort den grössten Nutzen stiftet, wo viele repetitive Routinetätigkeiten anfallen. Berufe mit viel Routine sind gerade in den mittleren Einkommensgruppen stark vertreten. Die fortschreitende Automatisierung könnte daher den Mittelstand ausdünnen und zu einer Polarisierung der Arbeitswelt beitragen. Tatsächlich haben die Beschäftigungsanteile von hoch und niedrig bezahlten Berufen von 1993 bis 2010 merklich zugenommen, während der Anteil der durchschnittlich entlohnten Berufe deutlich schrumpfte. Die Forscher schätzen, dass eine Zunahme der Routineintensität um eine Standardabweichung das Beschäftigungswachstum in den exponierten Berufen mit mittlerem Einkommen um 0,9 Prozentpunkte verlangsamte. Sie betonen, dass vor allem der technologische Fortschritt und weniger die Globalisierung die Ausdünnung des Mittelstands und die Polarisierung der Arbeitswelt prägt.

Was sich heute als Herausforderung der Automatisierung und Digitalisierung neu stellt, hat sich bereits in der IT-Revolution der 90-er Jahre gezeigt. Von 2002 bis 2012 ist in den U.S.A. der Lohn der untersten $10 \%$ der Lohnskala über 10 Jahre um nur 9,5\% gestiegen, während die obersten $10 \%$ einen Anstieg um 
22,5\% verbuchen konnten. Der vierte Beitrag zeigt, wie der Anstieg der Bildungsprämie von der Bildungs- und Wissensintensität des technologischen Fortschritts abhängt. Die Produktion verlangt zunehmend hochqualifizierte Arbeit, während weniger gut ausgebildete Arbeitskräfte teilweise wegrationalisiert werden. Die Wissenschaftler schätzten, dass alleine der technologische Wandel mit seiner gegenseitigen Abhängigkeit von Kapital und Bildung isoliert betrachtet einen Anstieg der Bildungsprämie um ca. $60 \%$ verursachte. Die gleichzeitige Erhöhung des Angebots an qualifizierten Arbeitskräften konnte die Prämie wieder um etwa $40 \%$ senken und den starken Anstieg teilweise kompensieren. Demnach hat die Bildungsprämie im Saldo wesentlich moderater um $18 \%$ zugenommen. Es zeigt sich, dass eine fundierte Ausbildung und lebenslanges Lernen wichtiger denn je sind, um in einem sich rasch wandelnden Arbeitsmarkt nicht als Verlierer dazustehen.

Innovation ist schöpferische Zerstörung und begünstigt tendenziell die Talentierten, die Unternehmer und die reichen Eigentümer, die Risikokapital einsetzen. In den U.S.A. hat sich zwischen 1975 bis 2013 der Anteil der Top-1-Prozent der Spitzenverdiener am Gesamteinkommen von 8,8 auf 20,1\% mehr als verdoppelt. Die im fünften Beitrag zusammengefassten Forschungsergebnisse belegen, wie die Zunahme der Top-1-Prozent unter Berücksichtigung vieler anderer Einflussgrössen ursächlich mit zunehmender Innovation zusammenhängt. Demnach steigert eine um $10 \%$ höhere Anzahl von Patenten den Einkommensanteil der Spitzenverdiener um 2,4\%. Etwa $22 \%$ des Anstiegs des Einkommensanteils der Top-1-Prozent entfallen auf Innovation. Zunehmende Innovation hat dagegen kaum einen signifikanten Einfluss auf die Verteilung im Rest der Bevölkerung. Bemerkenswert ist zudem, dass Innovationen vor allem in neu gegründeten Unternehmen die Aufstiegschancen verbessern und die soziale Mobilität erhöhen. Marktabschottung durch etablierte Unternehmen dagegen zementiert den Reichtum und bremst die soziale Mobilität.

Die Industrie ist ein Treiber der Innovation und des Produktivitätswachstums. Viele beklagen eine fortschreitende Deindustrialisierung. Was bedeutet eigentlich Deindustrialisierung genau und wie bewältigen die Unternehmen den Strukturwandel? Das erörtert der sechste Beitrag am Beispiel Dänemarks, wo die Zahl der Arbeitsplätze in der Industrie seit 1986 um mehr als $40 \%$ abnahm. Deindustrialisierung bedeutet aber nicht automatisch das Ende der betroffenen Unternehmen. Die Forscher dokumentieren, dass $10 \%$ der Industriefirmen von 2002 bis 2007 in industrienahe Dienstleistungsbranchen wechselten, was $42 \%$ der Arbeitsplatzverluste in der Industrie erklärt. Unternehmen, welche die Branche wechselten, waren bereits vor dem Wechsel produktiver und beschäftigten mehr gut ausgebildete Mitarbeiter als jene, die in der Industrie verblieben. Zwei Jahre nach 
dem Branchenwechsel waren $53 \%$ der Belegschaft ausgetauscht, wobei sich der Anteil hoch qualifizierter Mitarbeiter verdoppelte. Diese Ergebnisse legen nahe, dass eine defensive Wirtschaftspolitik, welche einen hohen Industrieanteil erhalten will, gerade die agilen Unternehmen verfehlen würde, welche mit innovativen Dienstleistungen profitablere Geschäfte erschliessen und am Ende ihren Arbeitnehmern bessere Perspektiven bieten können.

\section{Banken, Finanzierung und Krisenrobustheit}

Jede Investition braucht eine Finanzierung, und die Sparer suchen den richtigen Kompromiss zwischen Ertrag und Risiko. Wie finden die Ersparnisse und Anlagevermögen zu ihrer besten Verwendung? Viele Anleger wollen Sicherheit, aber die Investitionen der Wirtschaft sind riskant. Die Sparer wollen ihr Geld kurzfristig verfügbar haben, aber die Unternehmen brauchen langfristig gebundene Finanzierung. Die Sparer verfügen oft nur über kleine Beträge, aber die Wirtschaft braucht Investitionsfinanzierung in grossem Stil. Die Sparer haben oft wenig Zeit und andere Prioritäten, aber es braucht Überwachung und Kontrolle, um den erwarteten Ertrag zu sichern. Ein leistungsfähiger Finanzplatz bringt die Ansprüche der Anleger mit den Bedürfnissen der Wirtschaft zusammen, damit Investition, Wachstum und Zukunftsvorsorge gelingen. Banken und die anderen Finanzakteure bauen Risiken durch Diversifikation ab. Sie verwandeln kurzfristig abrufbare Ersparnisse in langfristige Kreditfinanzierung um. Sie bündeln viele kleine Sparbeträge für grosse Finanzierungen. Sie machen die Investitionen mit den grössten Chancen auf Wachstum und Wertsteigerung ausfindig und leisten Überwachung und Kontrolle im Auftrag ihrer Kunden, die oft weder die Zeit noch die nötige Expertise haben.

Ein leistungsfähiger Finanzplatz stützt auf vielfache Weise das Wachstum. Indem er das Kapital auf die ertragreichsten Verwendungen lenkt und von schrumpfenden Unternehmen und Branchen abzieht, leistet er einen wesentlichen Beitrag zur Produktivität. Im Auf und Ab der Konjunktur und im laufenden Strukturwandel durch Innovation sind die Unternehmen und die Erträge der Finanzanlagen einem hohen gesamtwirtschaftlichen Risiko ausgesetzt. Dazu kommt das Risiko im Wettbewerb um Marktanteile im In- und Ausland, wo das starke und manchmal sogar spektakuläre Wachstum der innovativen und erfolgreichen Unternehmen sich im Schrumpfen bis hin zur Insolvenz der weniger leistungsfähigen Konkurrenten spiegelt. Mit systematischer Diversifikation und einer Glättung von Risiken über die Zeit durch Ausnutzung von Kapitalpuffern und Reserven kann ein leistungsfähiger Finanzplatz Risiken in grossem Stil abbauen und wirtschaftliche Sicherheit ermöglichen. Damit jedoch Banken und die anderen Finanzakteure tatsächlich als Stossdämpfer dienen und die Konjunktur stabilisieren können, müssen sie selbst 
über genügend risikotragendes Eigenkapital und hohe Liquiditätsreserven verfügen. Fehlt es an diesen Kapitalreserven, dann kann der Finanzsektor sich schnell zum Krisenherd wandeln und in eine eskalierende Finanzkrise schlittern, der die Wirtschaft destabilisiert. Die folgenden Beiträge belegen die Rolle der Banken und des Finanzplatzes für die Entwicklung der Gesamtwirtschaft mit empirischer Evidenz.

$\mathrm{Zu}$ Beginn der Krise der Eurozone war die geringe Eigenkapitalausstattung der Banken und die starke Abhängigkeit von Bankkrediten ein wesentlicher krisenverschärfender Faktor, während in den USA der Kapitalmarkt stark zur Finanzierung der Wirtschaft beitrug und die Abhängigkeit von Bankkrediten viel geringer war. Eine solche Situation steigert die Ausfallswahrscheinlichkeit, die Höhe der anfallenden Verluste, und die Ansteckungsgefahren der Banken untereinander. Der erste Beitrag präsentiert Schätzungen, wonach das systemische Risiko, das von einer einzelnen Bank ausgeht, in der Eurozone wesentlich höher als in den U.S.A. war. Bezogen auf das Jahr 2011 hätte demnach in einer Immobilienkrise eine Bank mit einer Bilanzsumme von 1 Bio. $€$ (die Deutsche Bank hatte eine Bilanzsumme von 1,6 Bio. €) ein systemisches Risiko von 78 Mio. $€$ dargestellt, in den U.S.A. dagegen nur von 48 Mio. €. Im Zuge einer scharfen Immobilienkrise wären die Wachstumsverluste in einem Land mit hoher Abhängigkeit von Bankenkrediten wesentlich höher als in einem Land mit stark ausgebauter Kapitalmarktfinanzierung. Die Forscher führen diese Entwicklungen auf die implizite Staatsgarantie und auf die schwache Kapitalisierung der Banken zurück. Inzwischen hat sich die Situation mit den stark verschärften Kapital- und Liquiditätsanforderungen und der Bankenunion mit den Überwachungs- und Abwicklungsmechanismen wesentlich gebessert. Zudem will die EU mit der Kapitalmarktunion die Rolle der Kapitalmärkte ausbauen und in Hinkunft eine ausgewogenere Mischung über Banken- und Kapitalmarktfinanzierung erreichen.

Was macht eine Bank krisenanfällig und wieviel trägt sie zur Ansteckung des ganzen Sektors bei? Die zweite Arbeit zeigt, dass in einer Krise die Ansteckungsgefahren zwischen Banken vor allem auf zwei Wegen entstehen, Interbankenkredite und Notverkäufe von Vermögenswerten, die einen Preiseinbruch und damit Vermögensverluste bei anderen Banken auslösen. Die Systemrelevanz einer Bank hängt nicht nur von ihrer Grösse und ihrer Verschuldung, sondern auch von der Verwundbarkeit ihrer Bilanz gegenüber einem Preisverfall von Vermögenswerten und ihrer Verflechtung mit anderen Banken ab. Zum Beispiel kann ein Schuldenschnitt bei Staatsanleihen hohe direkte Vermögensverluste im Bankensektor auslösen. Wenn nun aber die Banken Notverkäufe tätigen und eine Abwärtsspirale bei den Anleihepreisen auslösen, entstehen weitere Verluste, die bis zu sechs Mal so hoch sein können wie der ursprüngliche Schuldenschnitt. Eine Begrenzung des Verschuldungsgrads und eine gezielte Rekapitalisierung der Banken mit der 
grössten Systemrelavanz können eine Ausbreitung der Krise am wirksamsten verhindern.

Die Kreditwürdigkeit des Staates ist begrenzt. Die regulatorische Bevorzugung der Staatsanleihen macht die Banken gegenüber dem staatlichen Kreditrisiko verwundbar. Riskante Staatsanleihen werfen hohe Risikoprämien ab, müssen aber von den Banken anders als z. B. Unternehmenskredite nicht mit Eigenkapital unterlegt werden. Deshalb sind gerade in den Krisenstaaten der Eurozone die Staatsanleihen wegen ihrer hohen Zinsen für die dortigen Banken besonders interessant. Der dritte Beitrag zeigt, dass die Banken in den Krisenstaaten tatsächlich inländische Staatsanleihen zweimal so schnell angehäuft haben wie in den stabilen Staaten, wobei vor allem schwach kapitalisierte Banken eher Staatsanleihen erwerben anstatt Unternehmenskredite zu vergeben. Die Forscher schätzten, dass in den Krisenstaaten Kursverluste von $17 \%$ bei den Staatsanleihen, die mit einem Anstieg der Risikoprämien einhergehen, das Wachstum der Unternehmenskredite um bis zu 1,4 Prozentpunkte dämpften. Eine Staatsschuldenkrise beeinträchtigt also über den Bankensektor die Realwirtschaft.

Die bessere Eigenkapitalausstattung im Zuge der strengeren Kapitalanforderungen haben die Widerstandsfähigkeit der Banken gestärkt und die Wahrscheinlichkeit von Finanzkrisen in Europa reduziert. Wie der vierte Beitrag belegt, kommt es für die Stabilisierung der Wirtschaft sehr darauf an, wann die Banken ihr Eigenkapital aufstocken müssen. Im Boom sind die Gewinne gross und die Banken können auch leicht neues Eigenkapital auf dem Markt aufnehmen, in der Krise ist es angesichts der auftretenden Verluste fast unmöglich. Deshalb sind antizyklische Kapitalpuffer, also höhere Eigenkapitalanforderungen im Boom und geringere in der Rezession, für die Stabilisierung der Konjunktur so wichtig. Die Forscher schätzen, dass in der Hochkonjunktur eine Erhöhung von Eigenkapitalreserven um $0,1 \%$ zu einem Rückgang des Kreditangebots um 4 Prozentpunkte führt. Wegen der reichlichen Verfügbarkeit von Finanzierung im Boom bleiben negative Folgen für die Realwirtschaft weitgehend aus. Ein Aufbau der Eigenkapitalreserven um $1 \%$ vor einer Krise erhöht jedoch in der folgenden Rezession die Kredite um 9 Prozentpunkte, das Beschäftigungswachstum um 6 Prozentzpunkte und die Überlebenswahrscheinlichkeit der Unternehmen um 1 Prozentpunkt. Antizyklische Kapitalpuffer können also einen starken Beitrag zur Stabilisierung leisten.

In der richtigen Verfassung können also Banken einen wichtigen Beitrag zur Stabilisierung der Wirtschaft leisten. Aber nicht alle Banken sind gleich. Eine besondere Rolle spielen die Hausbanken. Sie sind lokal verankert, pflegen dauerhafte Geschäftsbeziehungen und kennen ihre Kunden ganz genau. Deshalb können sie auch in Krisenzeiten Kreditlinien weiterführen, während andere Geschäftsbanken 
weniger Geduld haben und Kredite schneller fällig stellen. Der fünfte Beitrag stellt Forschungsergebnisse vor, wonach die Hausbanken während einer Finanzkrise ihren Unternehmen ein um $30 \%$ höheres Kreditvolumen zur Verfügung stellen und einen um 0,12 Prozentpunkte niedrigeren Zins verrechnen als normale Geschäftsbanken. Die Wahrscheinlichkeit, dass ein Kredit nicht verlängert wird, ist bei normalen Geschäftsbanken um 6 bis 9 Prozentpunkte höher. Es zeigt sich auch, dass Hausbanken eine um 3 Prozentpunkte höhere Eigenkapitalquote haben. Ausgestattet mit diesem Puffer können sie auch während einer Krise die Kreditlinien weiterführen und ihre Kunden durch schwierige Zeiten lotsen.

Damit sich Innovation entfalten kann, muss Strukturwandel stattfinden. Dabei spielen Banken eine wichtige Rolle. Kreditwürdigkeitsprüfung, laufende Überwachung, und die Entscheidung, Kredite nicht mehr zu verlängern, steuern das Kapital von schrumpfenden hin zu rentablen Verwendungen mit besseren Wachstumsaussichten. Doch man muss die Banken machen lassen. Das zeigte sich gemäss dem sechsten Beitrag an den Auswirkungen einer umfassenden Reform des französischen Bankensektors im Jahr 1985, welche das staatlich gelenkte zu einem marktwirtschaftlich gesteuerten Bankensystem umstellte. Nach dieser Reform waren Banken weniger bereit, schlecht gehende Unternehmen am Leben $\mathrm{zu}$ halten und lenkten die Kreditvergabe energischer auf profitable und expandierende Unternehmen. Sie stutzten die Verschuldung der besonders bankabhängigen Unternehmen von $79 \%$ auf eine gesündere Quote von $69 \%$ nach der Reform zurecht, so dass die Eigenkapitalausstattung der Unternehmen um 8 Prozentpunkte stieg. Die Deregulierung führte zu einem Rückgang der Bilanzsumme in den besonders bankabhängigen und überfinanzierten Sektoren relativ zu anderen um $6 \%$. Dafür stiegen die Markteintritte und die neu geschaffenen Vermögenswerte um $26 \%$ stärker als in anderen, weniger bankabhängigen Branchen. Die Kreditentscheidungen der Banken haben also einen erheblichen Einfluss auf den Prozess der schöpferischen Zerstörung, der die Produktivität und das innovationsgestützte Wachstum stärkt.

Eine sorgfältige Kreditwürdigkeitsprüfung ist bei Unternehmen und Haushalten gleichermassen notwendig. Das zeigt der siebte Beitrag zu Zahltagskrediten in den U.S.A. Solche Kleinkredite über wenige Wochen ohne Sicherheiten helfen, vorübergehende Mehrausgaben oder unerwartete Einnahmenausfälle bis zum nächsten Gehaltseingang zu überbrücken, sind jedoch sehr teuer. Ein erleichterter Zugang zu Zahltagskrediten steigert die Wahrscheinlichkeit um 5,3 Prozentpunkte, dass ein Haushalt in Schwierigkeiten gerät. Während ein Viertel der Kreditnehmer ein bis zweimal im Jahr einen Kleinkredit aufnimmt, benutzen rund $30 \%$ mindestens zwölf Kredite pro Jahr. Die Forscher schlussfolgern, dass ein Konsumentenschutz mit Mass notwendig ist, damit nicht einzelne Anbieter die Unwissenheit und mangelnde Selbstkontrolle der Kunden ausnutzen. 
Legt eine langanhaltende Niedrigzinsphase den Keim für die nächste Finanzkrise? Niedrige Zinsen stauchen die Zinsspanne und mindern die Profitabilität des Bankensektors. Zudem gehen sie mit hoher Liquidität einher und ermöglichen selbst den schlecht kapitalisierten Banken noch eine günstige Refinanzierung. Der letzte Beitrag zeigt die Folgen für Volumen und Qualität der Kreditvergabe. Demnach steigt nach einer Senkung der Tagesgeldzinsen um 1 Prozentpunkt bei schlechter kapitalisierten Banken die Wahrscheinlichkeit einer Kreditzusage an zahlungssäumige Gläubiger um 3 Prozentpunkte mehr als bei stabilen Banken. Zudem weiten sie ihr riskantes Kreditvolumen um $18 \%$ mehr aus als die stabilen Banken. Banken mit wenig Eigenkapital vergeben riskantere Kredite, die mit einer um 5 Prozentpunkte höheren Wahrscheinlichkeit ausfallen. Gleichzeitig sinken die Anforderungen an die Besicherung. Die Wahrscheinlichkeit, dass diese Kredite gänzlich unbesichert bleiben, steigt im Vergleich zu gut kapitalisierten Banken um fast 7 Prozentpunkte. Über längere Zeit erhöht ein niedriger Leitzins nicht nur das Kreditvolumen, sonder steigert auch das Risiko des Kreditportfolios und den Umfang der faulen Kredite, vor allem bei verwundbaren Banken mit geringer Eigenkapitalausstattung. Das könnte der Keim für die nächste Krise sein.

\section{Fazit}

Die Volkswirtschaftslehre hält eine fast unüberschaubar grosse Fülle empirischer Forschungsergebnisse bereit, die es für die Politik und die Öffentlichkeit zu nutzen gilt. Dieser Band enthält nur ein kleine, aber hoffentlich wichtige Auswahl von Beiträgen. Die empirische Forschung diszipliniert die volkswirtschaftliche Theoriebildung, deren Einsichten die Chancen auf bessere wirtschaftspolitische Konzepte steigern. Auch in der volkwirtschaftlichen Theoriebildung findet dabei ein Prozess kreativer Zerstörung statt. Jene Theorien, die empirisch gut abgesichert sind, setzen sich durch und gewinnen an Glaubwürdigkeit und Einfluss. Was nicht gut abgestützt ist oder gar im Widerspruch zu empirischen Forschungsergebnissen steht, verliert über kurz oder lang an Bedeutung und scheidet aus.

Die empirische Forschung liefert der Wirschaftspolitik die grösstmögliche Sicherheit über die quantitativen Auswirkungen von Reformen, damit eine evidenzbasierte Politik möglich wird. Eine absolute Sicherheit kann es nicht geben. Zudem ändern sich einmal ermittelte Zusammenhänge über die Zeit, wenn die Wirtschaft ihre Struktur verändert oder wenn die institutionellen Rahmenbedingungen angepasst werden, und müssen durch neue Gesetzmässigkeiten ersetzt werden. Nur eines ist gewiss: ohne empirische Forschung gibt es mit Sicherheit keine evidenzbasierte Politik. Dann ist die Gefahr gross, dass die Folgen von wirtschaftspolitischen Massnahmen zum Gegenstand von Spekulation werden und der Erfolg der Politik hauptsächlich von Zufälligkeiten abhängt. 
Die Ergebnisse der Volkswirtschaftslehre sollen Nutzen nicht nur für die wirtschaftspolitischen Entscheidungsträger stiften, sondern auch für die Öffentlichkeit, die für eine wirksame demokratische Kontrolle ein unabhängiges Bild von wirtschaftlichen Zusammenhängen und von den Folgen der Politik braucht. Deshalb ist es wichtig, dass komplexe Zusammenhänge auf das Wesentliche vereinfacht und allgemein verständlich vermittelt werden. Das ist der wichtige Beitrag der Studierenden im Projekt Next Generation, die mit den vorliegenden Beiträgen die interessierte Öffentlichkeit an ihrem Studium teilhaben lassen.

Open Access Dieses Kapitel wird unter der Creative Commons Namensnennung 4.0 International Lizenz (http://creativecommons.org/licenses/by/4.0/deed.de) veröffentlicht, welche die Nutzung, Vervielfältigung, Bearbeitung, Verbreitung und Wiedergabe in jeglichem Medium und Format erlaubt, sofern Sie den/die ursprünglichen Autor(en) und die Quelle ordnungsgemäß nennen, einen Link zur Creative Commons Lizenz beifügen und angeben, ob Änderungen vorgenommen wurden.

Die in diesem Kapitel enthaltenen Bilder und sonstiges Drittmaterial unterliegen ebenfalls der genannten Creative Commons Lizenz, sofern sich aus der Abbildungslegende nichts anderes ergibt. Sofern das betreffende Material nicht unter der genannten Creative Commons Lizenz steht und die betreffende Handlung nicht nach gesetzlichen Vorschriften erlaubt ist, ist für die oben aufgeführten Weiterverwendungen des Materials die Einwilligung des jeweiligen Rechteinhabers einzuholen.

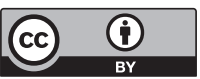

